\title{
CHEMSEX Y CONDUCTAS SEXUALES DE RIESGO EN HOMBRES QUE TIENEN SEXO CON HOMBRES: UNA REVISIÓN SISTEMÁTICA
}

\section{CHEMSEX AND RISKY SEXUAL BEHAVIOR IN MEN WHO HAVE SEX WITH MEN: A SYSTEMATIC REVIEW}

\author{
Itallo Carvalho Gomes, Mario Enrique Gámez-Medina, Carolina Valdez-Montero \\ Facultad de Enfermería Mochis, Universidad Autónoma de Sinaloa. México
}

\section{Abstract}

The term chemsex is used to talk about the sexualized use of drugs among men who have se with men (MSM) who feel the need to increase their arousal, pleasure and their duration in sexual intercourse. Objective. The objective of this work is to systematically analyze the available evidence on chemsex and risky sexual behaviors in MSM. Method. For the elaboration of this systematic review, the formulation of a question was followed, establishment of inclusion and exclusion criteria, development of search strategies, selection of articles to be included in the review, data extraction and data synthesis. Results. About chemsex, $25 \%$ of the studies describe how recreational drug use before or during the sexual encounter and $12.5 \%$ report that chemsex implies potential risks to the physical and mental health of its practitioner. $62.5 \%$ of studies associate this practice group sex parties. $62.5 \%$ of the studies found that homosexuals practice chemsex to a greater extent compared to bisexuals. Conclusions. This systematic review contributes to a greater understanding of chemsex and sexual risk behaviors among MSM.

Keywords: Chemsex, sexualized use of drugs, risk behaviors, gay men, Men who have sex with men.

\section{Resumen}

El término chemsex es utilizado para hablar del uso sexualizado de drogas entre los hombres que tienen sexo con hombres (HSH) que sienten la necesidad de incrementar su excitación, placer y su tiempo de duración en las relaciones sexuales. Objetivo. El objetivo del presente trabajo es analizar de manera sistemática la evidencia disponible sobre el chemsex y conductas sexuales de riesgo en HSH. Método. Para la elaboración de esta revisión sistemática se siguió con la formulación de una pregunta, establecimiento de criterios de inclusión y exclusión, desarrollo de estrategias de búsqueda, la selección de artículos para ser incluidos en la revisión, extracción de datos y síntesis de los datos. Resultados. Sobre el chemsex el $25 \%$ de los estudios describe el uso recreativo de drogas antes o durante el encuentro sexual y el $12.5 \%$ relata que el chemsex implica potenciales riesgos para la salud física y mental de su practicante. El $62.5 \%$ de los estudios asocian esa práctica a las fiestas de sexo en grupo. El $62.5 \%$ de los estudios encontraron que los homosexuales practican en mayor medida el chemsex comparado con los bisexuales. Conclusiones. La revisión sistemática contribuye a una mayor comprensión acerca del chemsex y las conductas sexuales de riesgo en $\mathrm{HSH}$.

Palabras clave: Chemsex, uso sexualizado de drogas, conductas de riesgo, hombres gay, hombres que tienen sexo con hombres. 
El término chemsex es utilizado para hablar del uso sexualizado de drogas entre los hombres que tienen sexo con hombres (HSH) que sienten la necesidad de incrementar su excitación, placer y su tiempo de duración en las relaciones sexuales (Tomkins, George \& Kliner, 2018). En la actualidad, el uso de redes sociales como sitios y aplicaciones (APPs) pueden facilitar la práctica de chemsex y a su vez posibilitar el encuentro con parejas sexuales (Gourlay et al, 2017). El desarrollo de APPs y sitios de geolocalización posibilita el acceso más rápido a prácticas sexuales con múltiples parejas, así como la facilidad de obtener drogas, lo cual a través del uso de la APPs permite negociar encuentros sexuales bajo efectos de alguna droga como estimulante a los encuentros sexuales con la excusa de que las drogas pudieran relajarlos y hacer que las relaciones sexuales fuesen más placenteras (Frankis \& Clutterbuck, 2017).

De este modo, al realizar la práctica del chemsex los HSH pueden utilizar diversos tipos de drogas recreativas como la metanfetamina cristalina, ácido gammahidroxibutita (GHB), gamma-butirolactona (GBL) y mefedrona (Tomkins et al, 2017), así como drogas inhalantes como los poppers (nitritos de alquilas) (Pepper, Zúñiga \& Reed, 2019). Los HSH practicantes del chemsex consideran tener una adicción a las drogas, particularmente aquellos que usan drogas inyectables, por lo tanto, rehúsan recibir tratamiento de rehabilitación (Frankis, \& Clutterbuck, 2017).

Por lo tanto, al hacer uso de drogas con la intención de relajar y potenciar la práctica sexual el practicante del chemsex se vuelve vulnerable debido al efecto de la droga, lo cual puede llevarlo a involucrarse con múltiples parejas sexuales, recibir dinero para mantener relaciones sexuales y compartir juguetes sexuales, lo que aumenta el riesgo de transmisión del $\mathrm{VIH}$ e Infecciones de transmisión sexual (ITS) (Tan et al, 2019). De acuerdo a la Organización Mundial de la Salud [OMS] (2017), 36.9 millones de personas en todo el mundo están infectadas con el VIH y señala que los HSH poseen 27 veces más probabilidades de infectarse con el virus. Por lo cual la práctica de chemsex es un problema de salud pública, debido a que existe un incremento en el número de personas involucradas en este comportamiento y un mayor riesgo sexual asociado a la práctica (Hibbert et al, 2019).
Por tal motivo, resulta importante conocer investigaciones respecto a la práctica del chemsex, así como los comportamientos sexuales de sus practicantes, dado que estudios recientes demuestran que los HSH son más propensos a usar drogas recreativas y son los más diagnosticados con el VIH en clínicas de salud sexual (Sewell et al, 2019). Por lo anterior, el objetivo del presente trabajo es analizar de manera sistemática la evidencia disponible sobre el chemsex y conductas sexuales de riesgo en $\mathrm{HSH}$.

\section{MÉTODO}

Para la elaboración de la revisión sistemática se siguieron los pasos propuestos por Holly, Salmond y Saimbert (2016): 1) Formulación de una pregunta, 2) Establecimiento de criterios de inclusión y exclusión, 3) Desarrollo de estrategias de búsqueda, 4) Selección de artículos para ser incluidos en la revisión, 5) Extracción de datos y 6) Síntesis de los datos. Primeramente para determinar la pregunta se utilizaron los componentes PEO (Población, Exposición, Outcomes/resultados) propuestos por Joanna Briggs (2014) para revisiones sistemáticas de estudios de etiología y riesgo. Estos componentes fueron: $\mathrm{P}=$ Hombres que tienen sexo con hombres, $\mathrm{E}=$ Chemsex y $\mathrm{O}=$ conductas sexuales de riesgo. Por lo tanto, la pregunta de la revisión sistemática fue ¿Cuál es la evidencia disponible sobre el chemsex y conductas sexuales de riesgo en $\mathrm{HSH}$ ?

Dentro de los criterios de inclusión de artículos se establecieron realizar una búsqueda de literatura entre enero de 2015 y marzo de 2019, debido al criterio de actualidad y por tratarse de una temática relativamente nueva, asimismo otros criterios fueron estar disponibles en idioma portugués, inglés o español; estar indexado; estudios con participantes HSH mayores de 16 años que realizaran la práctica chemsex y estar publicado como artículo original en revistas reconocidas. Los criterios de exclusión, fueron estudios repetidos en bases de datos. Respecto a la estrategia de búsqueda, se utilizaron bases de datos como MEDLINE, LILACS, PubMed y EBSCO, mediante el uso de los descriptores MeSh y DeCS como: "risk sexuality", "insecure sex", "male homosexuality", "chemsex". Además, se examinaron listas de referencias de los estudios encontrados como una forma de búsqueda manual. Se utilizaron algunas combinaciones 
de descriptores que fueron conectados con el operador booleano "AND". Como se muestra en la Tabla 1. La búsqueda de los estudios fue realizada en abril y mayo de 2019.

Tabla 1. Palabras clave conectadas con operadores booleanos usados en las búsquedas

\begin{tabular}{lc}
\hline $\begin{array}{l}\text { Descriptores con operadores } \\
\text { booleanos }\end{array}$ & $\begin{array}{c}\text { Cantidad de artículos } \\
\text { encontrados }\end{array}$ \\
\hline chemsex AND risk sexuality & 18 \\
chemsex AND condom & 46 \\
$\begin{array}{l}\text { insecure sex AND male } \\
\text { homosexuality }\end{array}$ & 32 \\
male homosexuality AND chemsex & 67 \\
\hline
\end{tabular}

Fuente: elaboración propia.

Se continuó con la selección de los artículos a través de la utilización de un checklist para estudios observacionales basado en el trabajo de Joanna Briggs (2014), la cual sirvió de apoyo para evaluar la calidad de los estudios. El checklist cuenta con criterios como: claridad del estudio, si se encuentra bien definida la problemática, si las personas que participaron de la investigación fueron seleccionadas de manera adecuada, si la probabilidad de sesgo fue presentada por los autores, también si los resultados de los estudios fueron concretos y si existe congruencia entre objetivos, hipótesis y pruebas estadísticas.
Para la extracción de datos, se realizó un esquema de codificación, usando una herramienta de los estudios descriptivos (observacionales) de Joanna Briggs (2014), para indicar las características generales de los estudios. Se formó una lista en la que se incluyó algunos componentes: autores, objetivos e hipótesis, año de publicación, país en que fue realizada la investigación, número de la población, edad de los participantes de los estudios, selección de los participantes, así como los resultados encontrados respecto a las variables de interés de cada estudio. Finalmente, para la síntesis de los hallazgos encontrados del chemsex y la conducta sexual de riesgo se realizó un resumen narrativo de cada uno de los estudios seleccionados recomendado por el manual Joanna Briggs (2014).

Se encontraron 163 artículos en las bases de datos y 5 artículos identificados a través de la referencia de un estudio, de los cuales fueron eliminados 65 estudios por duplicidad y 46 estudios por la lectura del título y el resumen debido a que no mostraban datos sobre chemsex o conducta sexual de riesgo. De los 11 artículos que permanecieron, 3 fueron eliminados por la evaluación metodológica. Finalmente quedaron 8 artículos incluidos para la revisión sistemática (Figura 1).
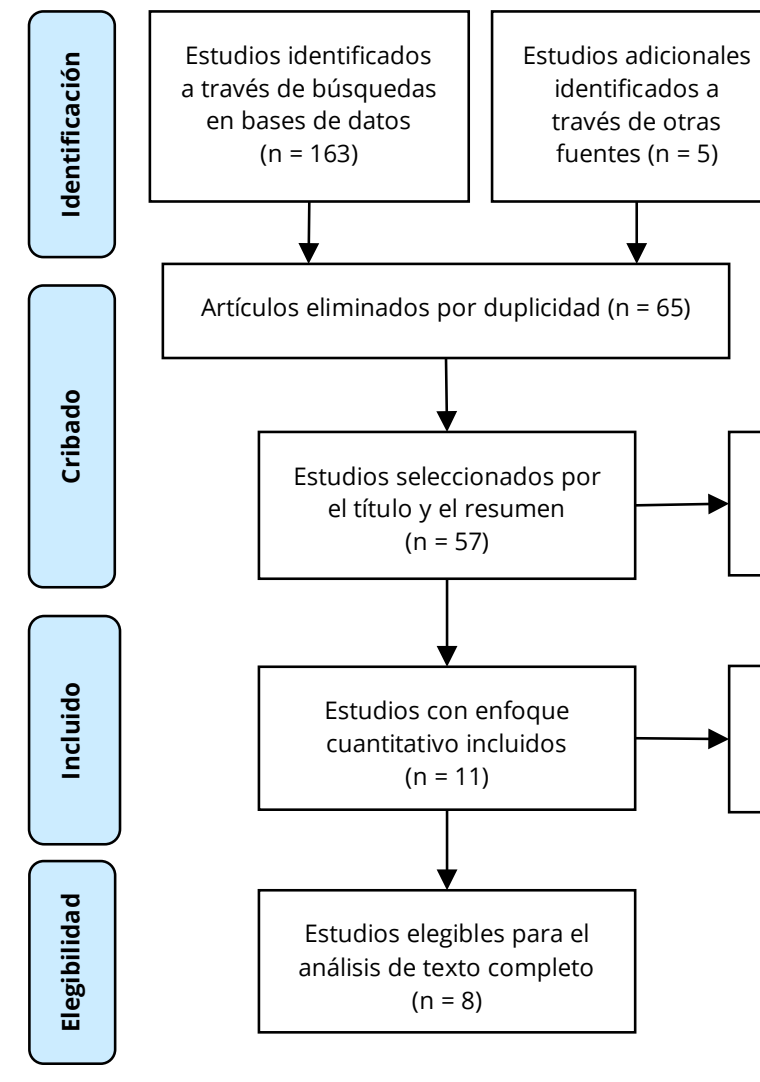

Estudios excluidos por el título y el resumen $(n=46)$

Eliminados por la evaluación metodológica $(n=3)$

Figura 1. Diagrama PRISMA 


\section{RESULTADOS}

En la Tabla 2 se muestran las características de los 8 estudios incluidos. Se encontró que el $50 \%$ de los estudios fueron realizados en la Inglaterra (Hegazi et al, 2016; Parry et al, 2019; Sewell et al, 2019; Tomkins et al, 2017). El 25\% en Reino Unido (Hibbert et al, 2019; Ottaway, Finnerty, Amlani, Pinto-Sander, Szanyi \& Richardson, 2016), y el 25\% fueron realizados en Francia e Irlanda (Glynn et al, 2018; Vaux et al, 2019). Respecto a la población participante en los estudios, el 50\% tuvieron una muestra mayor de 1,000 participantes (Hbbert et al, 2019; Parry et al, 2019; Sewell et al, 2019; Vaux et al, 2019), y el resto de los estudios tuvo una muestra menor de 500 participantes (Glynn et al, 2018; Ottaway et al, 2016; Tomkins et al, 2017; Tomkins et al, 2018). En cuanto a la media de edad de los participantes el $62.5 \%$ de las investigaciones tuvieron una media de edad de 30 años (Glynn et al, 2018; Hegazi et al, 2016; Sewell et al, 2019; Tomkins et al, 2017; Tomkins, George \& Kliner, 2018), el $25 \%$ de los estudios tuvieron una media edad de 40 años (Ottaway et al, 2016; Vaux et al, 2019) y el $12.5 \%$ una media de edad de 28 años (Parry et al, 2019).

Tabla 2. Características de los estudios incluidos

\begin{tabular}{|c|c|c|c|c|c|}
\hline $\begin{array}{l}\text { Autor y } \\
\text { año }\end{array}$ & Revista & País & $\mathrm{N}$ & Edad & Procedencia \\
\hline $\begin{array}{l}\text { Hegazi } \\
\text { et al. } \\
(2016)\end{array}$ & $\begin{array}{l}\text { International } \\
\text { Journal of STD } \\
\text { \& AIDS }\end{array}$ & Inglaterra & 655 & \pm 35 & $\begin{array}{c}\text { Clínica de } \\
\text { Salud sexual }\end{array}$ \\
\hline $\begin{array}{l}\text { Tomkins } \\
\text { et al } \\
\text { (2017) }\end{array}$ & $\begin{array}{l}\text { International } \\
\text { Journal of STD } \\
\text { \& AIDS }\end{array}$ & Inglaterra & 52 & \pm 32 & $\begin{array}{c}\text { Encuesta en } \\
\text { línea }\end{array}$ \\
\hline $\begin{array}{l}\text { Parry } \\
\text { et al } \\
(2017)\end{array}$ & $\begin{array}{l}\text { British HIV } \\
\text { Association }\end{array}$ & Inglaterra & 2971 & \pm 28 & $\begin{array}{c}\text { Encuesta en } \\
\text { línea }\end{array}$ \\
\hline $\begin{array}{l}\text { Sewell } \\
\text { et al. } \\
\text { (2019). }\end{array}$ & $\begin{array}{l}\text { Elsevier } \\
\text { International } \\
\text { Journal of Drug }\end{array}$ & Inglaterra & 1167 & \pm 34 & $\begin{array}{c}\text { Clínica de } \\
\text { Salud sexual }\end{array}$ \\
\hline $\begin{array}{l}\text { Vaux } \\
\text { et al. } \\
\text { (2019) }\end{array}$ & $\begin{array}{l}\text { BMC Infectious } \\
\text { Diseases }\end{array}$ & Francia & 2646 & \pm 45 & Locales gays \\
\hline $\begin{array}{l}\text { Hibbert } \\
\text { et al. } \\
(2019)\end{array}$ & $\begin{array}{l}\text { Sexually } \\
\text { Transmitted } \\
\text { Infections }\end{array}$ & Reino Unido & 3676 & \pm 30 & $\begin{array}{c}\text { Encuesta en } \\
\text { línea }\end{array}$ \\
\hline $\begin{array}{l}\text { Ottawa } \\
\text { et al. } \\
(2016)\end{array}$ & $\begin{array}{l}\text { International } \\
\text { Journal of STD } \\
\text { \& AIDS }\end{array}$ & Reino Unido & 260 & \pm 44 & $\begin{array}{l}\text { Clínica de } \\
\text { Salud }\end{array}$ \\
\hline $\begin{array}{l}\text { Glynn } \\
\text { et al. } \\
(2018)\end{array}$ & $\begin{array}{l}\text { International } \\
\text { Journal of Drug } \\
\text { Policy }\end{array}$ & Irlanda & 486 & \pm 32 & $\begin{array}{l}\text { Clínica de } \\
\text { Salud gay }\end{array}$ \\
\hline
\end{tabular}

Por otra parte, fue identificada la orientación sexual de los HSH, en el estudio de Hegazi et al. (2016), el 91\% de los participantes manifestaron ser homosexuales y el 9\% bisexuales, en Sewell et al. (2016), se encontró que el $94.5 \%$ eran homosexuales y el $5.5 \%$ bisexuales. A su vez, otro estudio mostró que el $83.6 \%$ eran homosexuales y el 16.4\% bisexuales (Vaux et al, 2019). Así mismo, el estudio de Hibbert et al. (2019), reporta que el $88 \%$ eran homosexuales y el $7 \%$ bisexuales. Se destaca que en la mayoría de los estudios los participantes se identifican como homosexuales y en una menor proporción como bisexuales.

En la Tabla 3 se muestran las drogas de consumo utilizadas en la práctica de chemsex, se encontró que en el 75\% de los estudios, los participantes señalaron el uso de la Mefedrona, Gamma-hidroxibutirato (GHB) / Gama butirolactona (GBL) y la Metanfetamina Cristal como las más comunes para esta práctica.

Tabla 3. Drogas de consumo en la práctica del chemsex en los estudios incluidos

\begin{tabular}{|c|c|c|c|c|c|c|}
\hline & $M$ & GHB-GBL & $P$ & $\mathrm{MCl}$ & C & $\mathrm{K}$ \\
\hline $\begin{array}{l}\text { Hegazi } \\
\text { et al. } \\
(2016)\end{array}$ & $69.3 \%$ & $56.4 \%$ & $\mathrm{NU}$ & $46 \%$ & $15.8 \%$ & $5.9 \%$ \\
\hline $\begin{array}{l}\text { Tomkins } \\
\text { et al } \\
(2017)\end{array}$ & $81 \%$ & $79 \%$ & NU & $36 \%$ & $31 \%$ & $38 \%$ \\
\hline $\begin{array}{l}\text { Parry } \\
\text { et al. } \\
(2017)\end{array}$ & NU & NU & NU & NU & NU & NU \\
\hline $\begin{array}{l}\text { Sewell } \\
\text { et al. } \\
(2019)\end{array}$ & $28.5 \%$ & $20.3 \%$ & NU & $10 \%$ & NU & NU \\
\hline $\begin{array}{l}\text { Vaux } \\
\text { et al. } \\
(2019)\end{array}$ & NU & UN & NU & NU & NU & NU \\
\hline $\begin{array}{l}\text { Hibbert } \\
\text { et al } \\
(2019)\end{array}$ & $3 \%$ & $3 \%$ & $28 \%$ & $2 \%$ & $2 \%$ & $10 \%$ \\
\hline $\begin{array}{l}\text { Ottaway } \\
\text { et al } \\
(2016)\end{array}$ & $20.8 \%$ & $20.8 \%$ & NU & $20.8 \%$ & $20.8 \%$ & $20.8 \%$ \\
\hline $\begin{array}{l}\text { Glynn } \\
\text { et al } \\
(2018)\end{array}$ & $16 \%$ & $57.3 \%$ & NU & $21.4 \%$ & $46.6 \%$ & $29.8 \%$ \\
\hline $\begin{array}{l}\text { ota: } \quad \mathrm{M}=1 \\
\text { utirolacton } \\
=\text { Ketamina }\end{array}$ & $\begin{array}{r}\text { efedrona } \\
P=\mathrm{Por} \\
\mathrm{JN}=\mathrm{No}\end{array}$ & $\begin{array}{l}\text { GHB=Ga } \\
\text { ers; } \mathrm{MC}= \\
\text { izó. }\end{array}$ & $\begin{array}{l}\text { nma-h } \\
\text { letanf }\end{array}$ & $\begin{array}{l}\text { roxibutir } \\
\text { amina }\end{array}$ & $\begin{array}{l}\text { o; } \quad \mathrm{GB} \\
\text { istal; }\end{array}$ & $\begin{array}{l}=\text { Gamma } \\
\text { Cocaína }\end{array}$ \\
\hline
\end{tabular}

\section{Chemsex}

Por lo que se refiere a la práctica de chemsex el $25 \%$ de los estudios la describe como el uso recreativo de drogas antes o durante el encuentro sexual (Tomkins et al, 2017; Vaux et al, 2019), asimismo el $62.5 \%$ de los estudios se refiere a esta práctica, como uso de drogas 
sexualizadas asociada a las fiestas de sexo en grupo (Glynn et al, 2018; Hegazi et al, 2016; Hibbert et al. 2019; Ottaway et al, 2016; Vaux et al, 2019). El 12.5\% refiere que el chemsex implica potenciales riesgos para la salud física y mental de su practicante (Parry et al, 2019). Por último el $62.5 \%$ de los estudios encontraron que los HSH que se identificaron como homosexuales practicaban en mayor medida el chemsex comparado con los que se identifican como bisexuales (Glynn et al, 2018; Hegazi et al, 2016; Hibbert el al, 2019; Sewell et al, 2019; Vaux et al, 2019).

\section{Conductas sexuales de riesgo}

La Tabla 4 muestra los estudios respecto a las conductas sexuales de riesgo entre los HSH que practican el chemsex. Se resalta que en el 75\% de los estudios, los HSH practicaron sexo anal sin condón (Glynn et al, 2018; Hegazi et al, 2016; Hibbert et al, 2019; Ottaway et al, 2016; Sewell et al, 2019; Tomkins et al, 2017). Igualmente con un $75 \%$ de los estudios, los HSH refirierieron tener múltiples parejas sexuales (Glynn et al, 2018; Hegazi et al, 2016; Hibbert et al, 2019; Sewell et al, 2019; Vaux et al, 2019). En este sentido, se encontró que el $62.5 \%$ de los estudios, los HSH tenían una media de 6-10 parejas sexuales en los últimos 12 meses (Glynn et al, 2018; Hegazi et al, 2016; Hibbert et al, 2019; Sewell et al, 2019; Vaux et al, 2019;). Además, el 37.5\% de los estudios indicó que los participantes realizaron prácticas de experimentación, como el fisting, que se refiere a la introducción parcial o total de la mano en recto (Hibbert et al, 2019; Sewell et al, 2019; Vaux et al, 2019), y el 25\% de los estudios los HSH indicaron tener sexo con el uso compartido de juguetes sexuales (Hegazi et al, 2016; Tomkins et al, 2017).

\section{Relación entre el chemsex y las conductas sexuales de riesgo en $\mathrm{HSH}$}

El total de los estudios muestran resultados de chemsex y conductas sexuales de riesgo, sin embargo, solo $87.5 \%$ de los mismos presentan una asociación entre las variables (Glynn et al, 2018; Hegazi et al, 2016; Hibbert et al, 2019; Ottaway et al, 2016; Sewell et al, 2019; Tomkins et al. 2017; Vaux et al, 2019;). Los HSH que practicaron chemsex tuvieron mayor probabilidad de desarrollar una ITS en comparación con los HSH que no lo practicaron (Hibbert et al., 2019).

Por su parte, el estudio de Hegazi et al (2016), reveló que los $\mathrm{HSH}$ que se involucraron en el chemsex fueron mayormente diagnosticados con ITS (48.7\%) como clamidia, gonorrea y sífilis comparado con los HSH que no practicaron chemsex (19.9\%). Además, se encontró que los practicantes de chemsex tenían mayor probabilidad de realizar prácticas sexuales de riesgo como sexo grupal, fisting y el uso compartido de juguetes sexuales en sus relaciones sexuales. El estudio de Ottaway et al. (2016), también señaló que los HSH que practican chemsex se asocian con adquisición de ITS, mayor prevalencia del VIH, mayor número de parejas sexuales y con prácticas sexuales anales sin uso del condón.

Tabla 4. Conductas sexuales de riesgo entre los HSH practicantes de chemsex

\begin{tabular}{|c|c|c|c|c|c|c|c|}
\hline & SASC & SOSC & SVSC & SG & MPS & JS & $\mathrm{FI}$ \\
\hline $\begin{array}{l}\text { Hegazi } \\
\text { et al. } \\
(2016)\end{array}$ & * & -- & -- & * & * & * & * \\
\hline $\begin{array}{l}\text { Tomkins } \\
\text { et al } \\
(2017)\end{array}$ & * & * & -- & * & -- & * & * \\
\hline $\begin{array}{l}\text { Parry et } \\
\text { al (2017) }\end{array}$ & -- & -- & -- & -- & -- & -- & -- \\
\hline $\begin{array}{l}\text { Sewell et } \\
\text { al. (2019) }\end{array}$ & * & -- & -- & * & * & -- & -- \\
\hline $\begin{array}{l}\text { Vaux et } \\
\text { al. (2019) }\end{array}$ & -- & -- & -- & -- & * & -- & * \\
\hline $\begin{array}{l}\text { Hibbert } \\
\text { et al } \\
(2019)\end{array}$ & * & -- & -- & -- & * & -- & -- \\
\hline $\begin{array}{l}\text { Ottaway } \\
\text { et al } \\
(2016)\end{array}$ & * & -- & -- & -- & * & -- & -- \\
\hline $\begin{array}{l}\text { Glynn et } \\
\text { al (2018) }\end{array}$ & * & -- & -- & -- & * & -- & -- \\
\hline
\end{tabular}

Nota: SASC= Sexo anal sin condón; SOSC= Sexo oral sin condón; SVSC= Sexo vaginal sin condón; SG= Sexo grupal; MPS= Múltiples parejas sexuales; JS= Juguetes sexuales; $\mathrm{FI}=$ Fisting.

\section{DISCUSIÓN}

Esta revisión sistemática tuvo como objetivo analizar de manera sistemática las evidencias disponibles sobre el chemsex y conductas sexuales de riesgo entre HSH. Se revisaron estudios con enfoque cuantitativo debido a la propuesta de analizar los hallazgos que asociaban las variables. Con relación a la colecta de datos de los estudios, estos fueron obtenidos en clínicas de salud y encuestas en línea donde cada uno de ellos cuido de la privacidad y el anonimato de los participantes. Todos los 
estudios abordaron población de HSH mayores de 28 años, con orientación homosexual y bisexual.

Los hallazgos encontrados en los estudios, referente a las drogas consumidas en la práctica del chemsex indican que los HSH utilizan más las drogas GHB/GBL, mefedrona y otros tipos de drogas como las inyectables. Lo anterior pudiera deberse a que después de su consumo el individuo comienza a sentir los efectos de forma inmediata y según la dosis los efectos pueden durar hasta 6 horas, creando un efecto prolongado el cual puede verse potencializado por la combinación con otras drogas que se toman, las cuales tienden a estimular la liberación de dopamina y bloquear su recaptación a través de su interacción con el transportador de dopamina, además de tener afinidad por varios subtipos de receptores de serotonina que causan un efecto de mejora temporal en la función mental y/o física con diferentes efectos emocionales y sociales (Giorgetti, Tagliabracci, Schifano, Zaami, Marinelli \& Busardò, 2017). Asimismo, Glynn et al. (2018), afirman que el uso de drogas como GHB/GBL en la práctica de Chemsex puede desencadenar la dependencia física de los usuarios, ya que es una droga de fácil acceso y bajo costo, por lo tanto, las personas tienden a usarlas frecuentemente $y$ en grandes cantidades.

Con respecto a chemsex, esta práctica se ha convertido en un problema creciente de salud pública en los países occidentales entre los HSH (Ottaway et al, 2016). Las razones por las cuales los hombres homosexuales y /o bisexuales se involucran en el uso de drogas durante el sexo aún son complejas, ya que cada persona puede tener una razón diferente para el uso de drogas sexualizadas (Glynn et al, 2018). Estos pueden estar relacionados con la búsqueda de sensaciones, nuevas experiencias sexuales e incluso inhibir la timidez o el cansancio físico. Hibbert et al. (2019), afirman que una de las razones del uso de drogas durante el sexo puede realcionarse con episodios previos de discriminación en algún momento de la vida, lo que puede causar tensión emocional en el individuo.

El índice de HSH que practican el chemsex y tienen sexo sin condón cuando están bajo el efecto de alguna droga es bastante alarmante (Hibbert et al, 2019; Tomkins et al, 2018). Además, de la práctica del fisting que se ha asociado estrechamente como una práctica de los $\mathrm{HSH}$, que puede causar un traumatismo rectal en la pareja y esto puede facilitar la transmisión de ITS / VIH cuando el pene penetra el ano sin condón (Rice, Maierhofer, Fields, Ervin, Lanza \& Turner, 2016). Además, que los HSH están utilizando cada vez más juguetes sexuales ya que estos juguetes se han hecho más populares y más fáciles de adquirir hoy en día debido a su comercialización en Internet (Döring \& Poesch, 2019). Los juguetes sexuales que más usan los HSH son los de estimulación anal como los "dildos" (prótesis de pene) y que se incorporan a la práctica sexual para que ambas partes sean participantes activos en el uso de juguetes convirtiéndose así en una práctica sexual arriesgada (Rosenberger et al, 2011).

Se identificó que la práctica del chemsex está completamente ligada a la incidencia de VIH e ITS pues una gran parte de los estudios reportó que la mayoría de Ios HSH eran VIH positivo y/o tenían una ITS y que en muchos de los casos no conocían de su condición actual tanto para el VIH como de ITS (Sewell et al, 2019; Vaux et al, 2019). Asimismo, las personas con alguna ITS o portadoras del VIH son más propensas a involucrarse en el uso sexualizado de drogas como también tener relaciones sexuales sin condón (Ottaway et al, 2016).

Los HSH que se involucran en la práctica del Chemsex lo hacen para que las drogas amplifiquen múltiples efectos en el cuerpo y por lo tanto, faciliten, aumenten y prolonguen el acto sexual, no obstante al buscar estos efectos, los practicantes de este acto pueden haber afectado su sentido conductual de asumir riesgos y en consecuencia, desencadenar efectos negativos como contraer el VIH o ITS en casos de penetración sin condón, sexo oral sin protección y también a través de lesiones anales causadas por fisting y juguetes sexuales compartidos (Maxwell, Shahmanesh \& Gafos, 2019).

\section{Conclusiones}

Sobre la práctica del chemsex, se conoce que el uso de drogas recreativas asociado a la práctica del sexo es bastante antiguo, pero el término chemsex es relativamente nuevo y encontrar estudios publicados con base en datos importantes que asocien esa práctica con las conductas sexuales de riesgo aún es limitada. Se buscó de forma integral el máximo de artículos que respondiera a la pregunta de partida para esta revisión sistemática, sin embargo, no todos los artículos encontrados proporcionaban información importante o 
relacionada a las conductas sexuales de riesgo bajo la práctica del chemsex, por lo que varios estudios fueron excluidos. Debido al número limitado de artículos incluidos y la falta de algunos datos estadísticos en ellos, los resultados obtenidos en este trabajo pueden tener algunas limitaciones.

Por lo tanto, debido a la escasez de artículos sobre un concepto claro sobre lo que es el chemsex y el daño que puede traer a la vida de sus practicantes es necesario realizar investigaciones adicionales en esa área para ayudar a responder de forma más clara y concisa lo que es el chemsex y cómo esta práctica puede ser sexualmente perjudicial para sus participantes.

Esta revisión sistemática contribuye a una mayor comprensión acerca del chemsex y de las conductas sexuales de riesgo, la cual puede ayudar a direccionar investigaciones futuras más específicas sobre la población HSH que practican el chemsex, así como de los riesgos físicos, biológicos y psicológicos a las que se exponen.

\section{Agradecimientos}

Al Consejo Nacional de Ciencia y Tecnología (CONACYT), el trabajo fue apoyado por la beca nacional de posgrados (PNPC: 005560) otorgado a Itallo Carvalho Gomes.

\section{REFERENCIAS}

Avinó, C. J. Olalla, P.G. Díez, E. Martín, S., \& Caylà, J.A. (2015). Explicaciones de las prácticas sexuales de riesgo en hombres que tienen sexo con hombres. Gaceta Sanitaria, 29(4), 252-257. doi:10.1016/j.gaceta.2015.03.002

Briggs, J. (2014).The Systematic Review of Prevalence and Incidence Data. Australia: Joanna Briggs Institute.

Döring, N., \& Poeschl, S. (2019). Experiences With Diverse Sex Toys Among German Heterosexual Adults: Findings From a National Online Survey. The Journal of Sex Research. doi:10.1080/00224499.2019.1578329

Evers, Y. J., Van Liere, G. A. F. S., Hoebe, C. J. P. A., \& Dukers-Muijrers, N. H. T. M. (2019). Chemsex among men who have sex with men living outside major cities and associations with sexually transmitted infections: A cross- sectional study in the Netherlands. Plos/One doi:10.1371/journal.pone.0216732

Frankis, F., \& Clutterbuck, D. (2017). What does the latest research evidence mean for practitioners who work with gay and bisexual men engaging in chemsex? Sexually Transmitted Infections, 93(3), 153-154. doi:10.1136/sextrans-2016-052783

Giorgetti, R., Tagliabracci, A., Schifano, F., Zaami, S., Marinelli, E., \& Busardò, F. (2017), When "Chems" Meet Sex: A Rising Phenomenon Called "ChemSex". Current Neuropharmacology, 15, 762-770. doi:10.2174/1570159X15666161117151148

Glynn, R. W., Byrnea, N., O'Deab, S., Shanleyb, A., Coddc, M., Keenand, E., Warda, M., Igoee, D., \& Clarkeb, S. (2018). Chemsex, risk behaviours and sexually transmitted infections among men who have sex with men in Dublin, Ireland. International Journal of Drug Policy, 52, 9-15. doi:10.1016/j.drugpo.2017.10.008

Gourlay, A. Fox, J. Gafos, M. Fidler, S. Nwokolo, N. Clarke, \& A. Gilson, R. (2017). A qualitative study exploring the social and environmental context of recently acquired HIV infection among men who have sex with men in South- East England. BMJ Open, 7:e016494. doi: 10.1136/bmjopen-2017-016494

Hibbert, M. P., Brett, C. E. Porcellato, L. A., \& Hope, V. D. (2019). Psychosocial and sexual characteristics associated with sexualised drug use and chemsex among men who have sex with men (MSM) in the UK. Sexually Transmitted Infections, 95(1), 342-350. doi:10.1136/sextrans-2018-053933

Holly, C., Salmond, S., \& Saimbert, M. (2016). Comprehensive Systematic Review for Advanced Practice Nursing. $2^{a}$ edic. New York: Springer Publishing Company.

Maxwell, S., Shahmanesh, M., \& Gafos, S. (2019). Chemsex behaviours among men who have sex with men: A systematic review of the literature. International Journal of Drug Policy, 63, 74-89. doi:10.1016/j.drugpo.2018.11.014

Organización Mundial de la Salud. (2017). VIH/sida. Consultado [27 de junio de 2019]. Recuperado de: https://www.who.int/es/newsroom/fact-sheets/detail/hiv-aids.

Ottaway, Z, Finnerty, F., Amlani, A. Pinto-Sander, N., Szanyi, J., \& Richardson, D. (2016). Men who have sex with men diagnosed with a sexually transmitted infection are significantly more likely to engage in sexualised drug use. International Journal of STD \& AIDS doi:10.1177/0956462416666753

Pepper, N. Zúñiga, M.L., \& Reed, M.B. (2019). Prevalence and correlates of "popper" (amyl nitrite inhalant) use among HIV-positive Latinos living in the U.S.-Mexico border region. Journal of ethnicity in substance abuse. doi:10.1080/15332640.2018.1540955

Parry, S. Et al. (2019). Psychological wellbeing and use of alcohol and recreational drugs: results of the British HIV Association (BHIVA) national audit 2017. HIV Medicine, 20(6), 424-427. doi:10.1111/hiv.12744

Rice, C. E., Maierhofer, C., Fields, K. S., Ervin, M., Lanza, S. T., \& Turner, A. N. (2016). Beyond Anal Sex: Sexual Practices of Men Who Have Sex with Men and Associations With HIV and Other Sexually Transmitted Infections. The journal of sexual medicine, 13(3), 374 382. doi:10.1016/j.jsxm.2016.01.001

Rosenberger, J. G., Reece, M., Schick, V., Herbenick, D., Novak, D. S., van der Pol, B., \& Fortenberry, J. (2011). Sexual behaviors and situational 
characteristics of most recent male-partnered sexual event among gay and bisexually identified men in the United States. Journal of Sexual Medicine, 8(11), 3040-3050. doi:10.1111/j.1743$\underline{6109.2011 .02438 . x}$

Sewell, J., Cambiano, V., Speakman, A., Lampe, F. C., Phillips, A., Stuart, D.,...Rodger, A. J. (2019). Changes in chemsex and sexual behaviour over time, among a cohort of MSM in London and Brighton: Findings from the AURAH2 study. International Journal of Drug Policy, 68, 54-61. doi:10.1016/j.drugpo.2019.03.021

Tan, R .K .J., Wong, C. M., Chen, M. I., Chan, Y. Y., Ibrahim, M. A. B., Lim, O. Z.,...Choong, B. C. H. (2019). Chemsex among gay, bisexual, and other men who have sex with men in Singapore and the challenges ahead. International Journal of Drug Policy, 61, 31-37. doi:10.1016/j.drugpo.2018.10.002

Tomkins, A. George, R., \& Kliner, M. (2018). Sexualised drug taking among men who have sex with men: a systematic review. Perspectives in Public Health, 139(1), 23-33. doi:10.1177/1757913918778872.

Tomkins, A. Vivancos, R. Ward, C., \& Kliner, M. (2017). How can those engaging in chemsex best be supported? An online survey to gain intelligence in Greater Manchester. International Journal of STD \& AIDS, 29(2), 128-134. doi:10.1177/0956462417719643

Vaux, S., Chevaliez, S., Saboni, L., Sauvage, C., Sommen, C., Barin, F.,...Velter, A. (2019). Prevalence of hepatitis $C$ infection, screening and associated factors among men who have sex with men attending gay venues: a cross-sectional survey (PREVAGAY), France, 2015. BMC Infectious Diseases, 19(315), 1-14. doi:10.1186/s12879019-3945-Z 